\title{
The Comparison of Chemiluminescent- and Colorimetric-detection Based ELISA for Chinese Hamster Ovary Host Cell Proteins Quantification in Biotherapeutics
}

Fengqiang Wang*, Dennis Driscoll, Daisy Richardson and Alexandre Ambrogelly

Bioprocess Development, Merck Research Laboratories, Union, NJ, USA

\begin{abstract}
Biologics manufacturing requires the clearance of Host Cell Proteins (HCPs) from recombinant therapeutic protein to acceptable low levels to ensure product purity and patient safety. To ensure adequate removal, a highly sensitive method, commonly in the form of Enzyme-Linked Immunosorbent Assay (ELISA), is necessary to quantify the HCPs amount in process intermediates and drug substance. We report the development of a chemiluminescent detection based ELISA (luminescent ELISA) in lieu of previously used colorimetric method (colorimetric ELISA) to improve assay sensitivity for the quantification of Chinese Hamster Ovary (CHO) HCPs in a monoclonal antibody product (mAb-A). For luminescent ELISA, Pierce Supersignal ELISA Femto was chosen as the substrate to replace colorimetric substrate TMB. The assay performance of luminescent and colorimetric ELISA was directly compared side-by-side. Our data show that luminescent ELISA has better signal/background ratio, broader linear range over logarithmic scales, and better linearity within the same linear range than colorimetric ELISA. Luminescent ELISA also demonstrates better lowend linearity, greater accuracy and precision. In addition, the Limit of Detection (LOD) and Limit of Quantification (LOQ) are significantly improved with luminescent ELISA as compared to colorimetric ELISA. In summary, luminescent ELISA is a more sensitive method and demonstrates superiority over colorimetric method for CHO HCP quantification.
\end{abstract}

Keywords: Host cell proteins; ELISA; Chemiluminescence; Chinese hamster ovary; Biotherapeutics; Monoclonal antibody

\section{Introduction}

Monoclonal antibodies (mAbs) have become a significant focus of the pharmaceutical industry due to their high specificity and their ability to engage a wide variety of targets $[1,2]$. While $\mathrm{mAb}$ therapeutics have been produced in a variety of genetically engineered host cell of non-human origin such as bacteria, yeast, plant, insect and mammalian cells, they are most commonly expressed in immortalized Chinese hamster ovary $(\mathrm{CHO})$ cell lines [3-5]. $\mathrm{CHO}$ is a robust host that offers high productivity and glycosylation patterns similar to those found in endogenous human antibodies. Harvest of therapeutic antibodies of interest is relatively straight forward since the recombinant product is often secreted in the media. However, the harvest also contains significant amounts of proteins originated from the host, namely host cell proteins (HCPs), which are either secreted during fermentation or released into culture fluid as a result of cell lysis. Due to their nonhuman origin and thus potential immunogenic nature, HCPs can pose significant safety risk for patients and are part of process-related impurities that need to be controlled during bioprocess development [6-8]. Since after the purification steps, the residual HCPs amount in final drug substance is often very low in the parts per million (ppm) level, a highly specific, highly sensitive, and quantitative assay is desired to ensure their adequate removal and patient safety $[9,10]$. Due to its high specificity and sensivity, enzyme-linked immunosorbent assay (ELISA) is the most commonly accepted method by regulators for HCPs quantification [10]. Alternative immunospecific methods such as a quantitative slot blot assay [11] and solid-phase proximity ligation assay [12] as well as non-specific methods including mass spectrometry (MS) and 2D liquid chromatography (LC)-MS are also being developed or explored $[8,10]$. However, none of these methods are robust enough or can achieve the same level of sensitivity as ELISA, which remains the gold standard for HCP quantification. Commercially available HCP ELISA kits, commonly used as generic HCP assays in the early phase of development (Phase I/II) as well as previously reported late stage process-specific HCP ELISA often use colorimetric detection for signal generation $[13,14]$. Colorimetric detection limits the assay sensitivity for low levels of HCP especially in final drug product $[13,15]$. At Merck Research Laboratories, we have initially developed a process-specific colorimetric ELISA assay for one of our late stage $\mathrm{CHO}$-produced monoclonal antibodies, mAb-A. Genetically engineered $\mathrm{CHO}$ cell line is used to manufacture mAb-A and thus a process-specific HCP ELISA using proprietary antibodies raised against the null $\mathrm{CHO}$ cells has been developed in-house for Phase III mAb-A to measure HCP components in the drug substance (DS).

The process-specific ELISA in its current format has a limit of quantification (LOQ) of $7.6 \mathrm{ng} / \mathrm{ml}$ in $5 \mathrm{mg} / \mathrm{ml}$ of drug substance (equivalent to $1.5 \mathrm{ppm}$ ). While this LOQ value is sufficient to demonstrate process clearance of HCPs, improvements can be made to increase the assay sensitivity to measure HCP concentration $<7.6$ $\mathrm{ng} / \mathrm{ml}$. Since colorimetric detection limits the assay sensitivity for low levels of HCPs especially in the final drug susbtance, alternative method using chemiluminescent detection has been explored. Since its introduction in the late 1970s, chemiluminescence has been used in a variety of analytical and immunological tests such as high performance liquid chromatography [16], capillary electrophoresis

${ }^{*}$ Corresponding author: Fengqiang Wang, Ph.D., Associate Principa Scientist, Bioprocess Development, Merck Research Laboratories, Kenilworth, NJ, USA, Tel: 908-740-5106; Fax: 908-740-4366; Email: fengqiang.wang@merck.com

Received September 12, 2013; Accepted October 21, 2013; Published October 30

Citation: Wang F, Driscoll D, Richardson D, Ambrogelly A (2013) The Comparison of Chemiluminescent- and Colorimetric-detection Based ELISA for Chinese Hamster Ovary Host Cell Proteins Quantification in Biotherapeutics. J Bioprocess Biotech 3: 136 doi: 10.4172/2155-9821.1000136

Copyright: ( 2013 Wang F, et al. This is an open-access article distributed unde the terms of the Creative Commons Attribution License, which permits unrestricted use, distribution, and reproduction in any medium, provided the original author and source are credited. 
[17], immunoassays and DNA analyses [18,19]. Analytical methods using chemiluminescent detection are often characterized by their high sensitivity, broader dynamic range, and high signal-to-noise ratio [19]. In immunoassays, chemiluminescent horse radish peroxidases (HRP) substrates have shown improved sensitivity over colorimetric substrates [20]. Commercially available Thermo Scientific Super Signal ELISA Femto Maximum Sensitivity substrate uses an improved enhancer system with much greater sensitivity and has been successfully used in high throughput enzyme immunoassay [21], antibody microarrays [22], and blood-based diagnostic assays [23]. With its known advantage of fast light generation, high sensitivity $(1.7 \mathrm{pg} / \mathrm{ml})$, and improved low-end linearity, the ELISA Femto substrate was adopted for assay development and its assay performance was compared side-by-side with 1-step turbo TMB (sensitivity $70 \mathrm{pg} / \mathrm{ml}$ ) based colorimetric detection method. The signal/noise ratio, linear range and linearity over logarithmic scales, precision and accuracy as well as the limit of detection (LOD) and limit of quantification (LOQ) of both methods were assessed and compared following ICH guidelines-Q2 (R1).

\section{Materials and Methods}

\section{Commercial reagents and consumables}

Hyclone phosphate buffer saline (PBS, 10×), carbonate-bicarbonate buffer packet PK40, blocker BSA in PBS (10×), neutrAvidin-horseradish peroxidase (HRP) conjugate, 1-step Turbo TMB ELISA substrate, and Supersignal ELISA Femto Maximum Sensitivity substrate were purchased from Thermo Fisher Scientific Inc.(Waltham, MA, USA); Tween-20 was purchased from Sigma Aldrich (St. Louis, MO, USA); Costar EIA/RIA ELISA clear bottom 96-well plate was purchased from Corning Inc (Corning, NY, USA); NUNC white opaque 96-well plate was purchased from Thermo Fisher Scientific Inc. (Waltham, MA, USA). ImmunoWare tubes and ImmunoWare reagent reservoirs were product of Thermo Scientific Pierce Inc (Waltham, MA, USA).

\section{Merck proprietary reagents}

Anti-HCP polyclonal antibodies were raised in goat by Pocono Farms\& Laboratory, Inc. (Tobyhanna, PA, USA) against CHO null cell culture (mock) that doesn't have the gene encoding $\mathrm{mAb}-\mathrm{A}$ and the anti-sera was affinity purified by a self-prepared mock HCP affinity column. Affinity-purified goat anti-HCP IgG was then aliquoted and partially labeled with biotin using EZ-link Sulfo-NHS-LC-Biotin kit from Thermo Scientific Inc (Waltham, MA, USA). Unlabeled antiCHO HCP antibody (lot\# 68780/140, $1.69 \mathrm{mg} / \mathrm{mL}$ ) was used as coating antibody and biotinylated goat anti-HCP IgG (lot\# 68780/147, 1.69 $\mathrm{mg} / \mathrm{mL}$ ) was used as detecting antibody in a sandwich ELISA format (Figure 1). CHO HCP stock generated from mock cell fermentation was used as standard $(5.70 \mathrm{mg} / \mathrm{mL}$, lot\# 68383/106). The reagents are stored at $-20^{\circ} \mathrm{C}$, with one working aliquot stored at $2-8^{\circ} \mathrm{C}$.

\section{Instruments}

ELISA plate wash was done using a BioTek Elx 405 Select semiautomatic plate washer (BioTek USA,Winooski, VT), and the absorbance/luminescence signal was read by a Molecular Devices SpectraMax M5 plate reader (Molecular Devices, Sunnyvale, CA).

\section{ELISA working solutions}

Coating buffer was prepared by dissolving one packet of the carbonate-bicarbonate buffer concentrate in $500 \mathrm{ml}$ of deionized water; washing solution was prepared by adding Tween-20 to $1 \times$ PBS to a final concentration of $0.1 \%$; blocking solution was prepared by adding
Tween-20 and $10 \times$ Pierce Blocker to PBS to a final solution containing $1 \%$ BSA and $0.05 \%$ Tween-20; assay diluents was prepared by adding Tween-20 and $10 \times$ Pierce Blocker to PBS to a final solution containing $0.1 \%$ BSA and $0.05 \%$ Tween-20.

\section{Performing CHO colorimetric ELISA and luminescent ELISA in Corning Costar clear EIA/RIA 96-well plate}

The CHO HCP ELISA was performed using established protocols for colorimetric detection with the adaption on the substrate addition step for chemiluminescent detection (Figure 1). For chemiluminescent ELISA, SuperSignal ELISA Femto substrate, instead of 1-step Turbo TMB substrate, was added to the appropriate wells and light emission was measured at $425 \mathrm{~nm}$. Briefly, the ELISA plate was coated by 100 $\mu \mathrm{L} /$ well of coating antibody solution $(1 \mu \mathrm{g} / \mathrm{ml})$ prepared in carbonatebicarbonate buffer and incubated at room temperature with gentle shaking for $2 \mathrm{hrs}$. After 4 washes with $300 \mu \mathrm{L} /$ well washing buffer (10 second incubation/wash) using BioTek Elx 405 Select plate washer, the plate was blot dried using tissue paper and incubated with $300 \mu \mathrm{L} /$ well of blocking solution at room temperature for $1 \mathrm{hr}$. After wash, the ELISA reactions were performed at room temperature with the addition of $\mathrm{CHO} \mathrm{HCP}$ standards or unspiked/spiked mAb-A samples (100 $\mu \mathrm{L} /$ well), followed by the subsequent incubation with biotinylated anti-CHO HCP antibody $(1 \mu \mathrm{g} / \mathrm{mL}, 100 \mu \mathrm{L} /$ well $)$, NeutrAvidin-HRP conjugate (1:15,000 dilution in assay diluents, $100 \mu \mathrm{L} /$ well), and 1-step TMB turbo ELISA substrate (100 $\mu \mathrm{L} /$ well $)$ or Supersignal ELISA Femto Maximum Sensitivity substrate $(100 \mu \mathrm{L} /$ well $)$. Plate was washed 4 times with $300 \mu \mathrm{L} /$ well washing buffer between each incubation steps. For those wells with TMB as a substrate, plate was incubated in dark for 10 min and the reactions were terminated by the addition of $100 \mu \mathrm{L} /$ well of $1 \mathrm{M}$ sulfuric acid, and then the absorbance of those wells were read at $450 \mathrm{~nm}$ using a Molecular Devices plate reader; for wells with ELISA Femto as a substrate, the light emission was measured at $425 \mathrm{~nm} 10 \mathrm{~min}$ after the addition of substrate using the same plate reader.

\section{Performing CHO HCP colorimetric and luminescent ELISA in Nunc opaque 96-well plate}

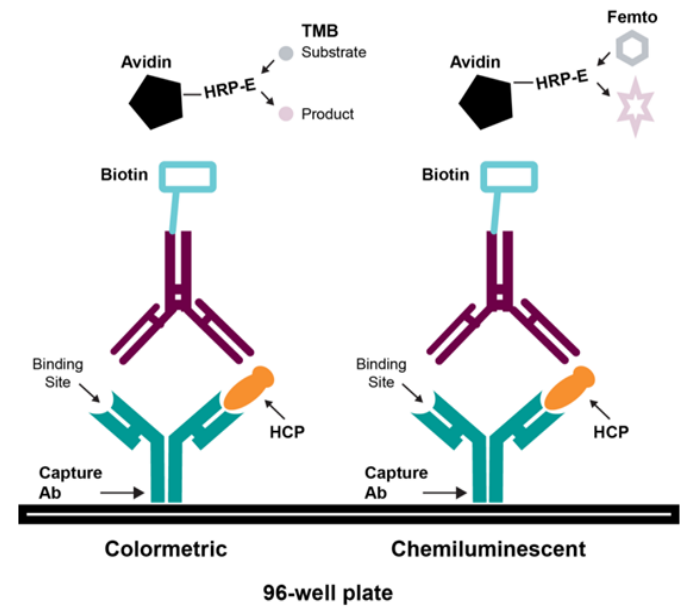

Figure 1: Schematic illustration of colorimetric and luminescent ELISA performed. Both ELISA methods are performed on the same 96-well plate and following the same procedure of HCPs capturing by coated anti-HCP antibodies, primary detection by biotinylated anti-HCP antibodies, and secondary detection by NeutrAvidin-HRP conjugates. The signal detection is either obtained by measuring absorbance at $450 \mathrm{~nm}$ generated from catalyzing TMB substrate (left) or by measuring the luminescence at $425 \mathrm{~nm}$ generated from oxidizing of ELISA Femto substrate (right). 
To avoid luminescence signal cross-interference from adjacent wells, NUNC white opaque 96-well plate that provides maximum reflection and low cross-talk was chosen for luminescent ELISA development and optimization. The ELISA procedure follows the same steps of coating, washing, blocking, standards and samples incubation, primary detection (biotin-antibody conjugate), secondary detection (NeutrAvidin-HRP conjugate), and substrate incubation steps as described previously. The ELISA Femto substrate solution was prepared fresh on day of use by mixing equal volume of the signal enhancer with the Femto substrate. The steps and reagent volumes used in each step are summarized in Supplemental Table 1. For colorimetric assay, the reaction was stopped with the addition of $1 \mathrm{M}$ sulfuric acid after 10 min incubation in TMB substrate, and then the reaction mixtures were transferred to a Costar clear 96-well plate using multi-channel pipette or and the absorbance of each well was measured at $450 \mathrm{~nm}$ using Molecular Devices SpectraMax microplate reader.

Standards and samples preparation for evaluating colorimetric and luminescent ELISA assay performance in Corning Costar 96-well clear plate

The ELISA assay performances including signal/noise ratio, dynamic range, low-end linearity, accuracy and precision were compared side-by-side using $\mathrm{CHO} \mathrm{HCP}$ standard in triplicate prepared in a series of 3-fold dilution over the range from $2000 \mathrm{ng} / \mathrm{ml}$ to $0.034 \mathrm{ng} /$ $\mathrm{ml}$. Additional experiments were performed using standards in a series of 2 -fold dilution ranging from 200 to $0.195 \mathrm{ng} / \mathrm{ml}$ or 50 to $0.049 \mathrm{ng} / \mathrm{ml}$. Different amount of CHO HCPs $(25 \mathrm{ng} / \mathrm{ml}, 5 \mathrm{ng} / \mathrm{ml}, 2 \mathrm{ng} / \mathrm{ml}$ and $1 \mathrm{ng} /$ $\mathrm{ml}$ ) were also spiked into $\mathrm{mAb}$-A drug substance (final concentration 5 $\mathrm{mg} / \mathrm{ml}$ ) to assess the accuracy and precision of each assay.

Standards and samples preparation for evaluating colorimetric ELISA and luminescent ELISA performance in NUNC white opaque 96-well plate

The ELISA assay performances including signal/noise ratio, the assay linearity, accuracy, precision, LLOD and LLOQ were compared over the standards range from $1.56 \mathrm{ng} / \mathrm{ml}$ to $100 \mathrm{ng} / \mathrm{ml}$ using the same amount of reagents (Supplemental Table 1) optimized for luminescent ELISA in NUNC white opaque plate.

\section{Data analysis}

All experiments were done in triplicate. The comparison of colorimetric and luminescent ELISA was performed side-by-side in three repeated experiments with slightly variation on standard range. The data analysis was performed using Molecular Devices Soft max Pro v 5.3 software and Microsoft Excel. The chemiluminescence light emission at $425 \mathrm{~nm}$ (E425) or absorbance values at $450 \mathrm{~nm}$ (A450) are plotted against standard concentrations using the software's built in 4-parameter fit, linear fit, or log-log curve fitting. The signal/noise ratio of each standard data point is determined using the signal generated by a standard at a given concentration to that of concentration 0 (blank). The linear range over logarithmic scales is determined as where the correlation coefficient (R2) has a value $>0.99$. The concentrations of standards are then back-calculated from the standard curve fit equation to assess the precision (CV\%) and accuracy (recovery\%) of the assay. In all conditions, the same curve-fitting method is applied for both colorimetric and luminescent ELISA for direct comparison. The recovery $\%$ of standards is calculated as the ratio of back-calculated concentration to the expected concentration $\times 100 \%$.

\section{Results and Discussion}

The assay performance of luminescent ELISA and colorimetric
ELISA was directly compared by a variety of assay parameters such as signal/noise ratio, linear range and linearity over logarithmic scales, accuracy and precision, lower limit of detection (LLOD) and quantification (LLOQ). To reduce the variations caused by assay plate type, the comparison of assay performance was carried out in both 96-well clear plate and opaque plate. Several standard curve-fitting methods built in the Softmax Pro v5.4.1 were applied to determine the optimum method for HCP quantification.

Assay performance comparison for colorimetric ELISA and luminescent ELISA in Corning Costar clear 96-well plate using conditions optimized for colorimetric ELISA

ELISA assays using 1-step Turbo TMB (colorimetric) or ELISA Femto (luminescent) as a substrate for $\mathrm{CHO}$ HCP testing were performed side-by-side on the same 96-well Costar clear EIA/RIA plate following the procedure described in materials and methods. The standard curves were first fit using a 4-parameter non-linear regression. As seen in Figure 2A, colorimetric ELISA standard curve (bottom) displayed a sigmoid shape with the absorbance value at 2000 $\mathrm{ng} / \mathrm{ml}$ reaching a plateau, in contrast, luminescent ELISA standard curve at $2000 \mathrm{ng} / \mathrm{ml}$ remains in the rising phase (top). The $\mathrm{C}$ values
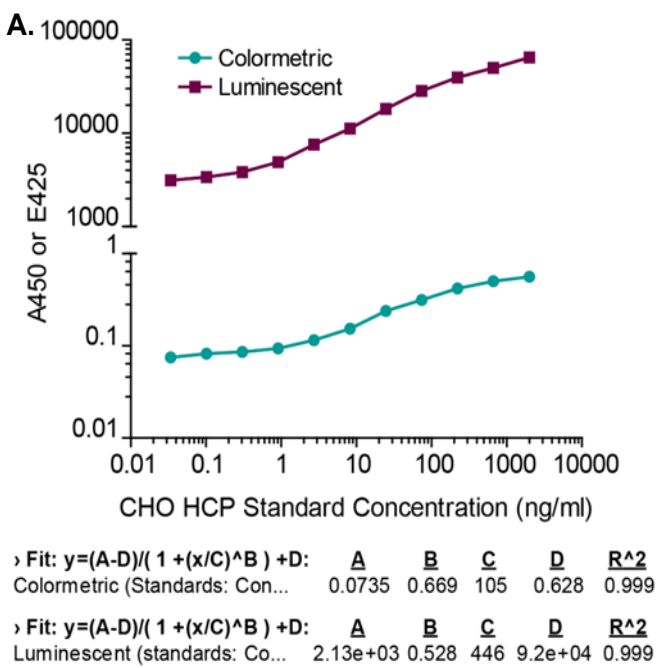

B.

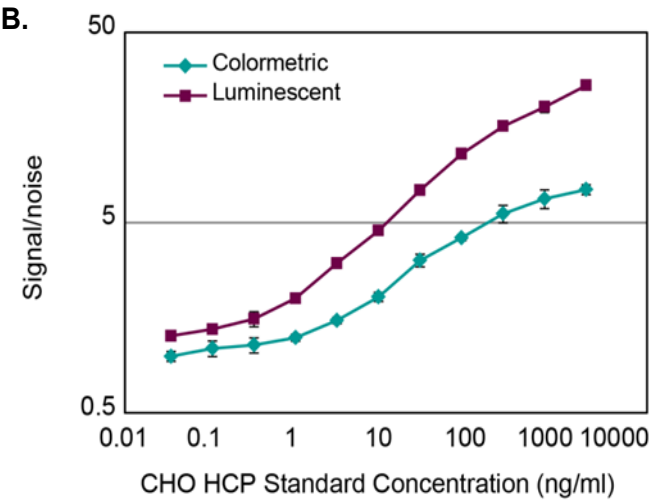

Figure 2: Standard curves from colorimetric and luminescent ELISA performed in clear 96-well plate. A. Absorbance values (A450) or luminescence emissions at $425 \mathrm{~nm}$ (E425) were plotted against standard concentrations on log$\log$ scales and the curve equations were fit using 4-parameter non-linear regression. B. Signal/noise ratio values plotted against $\mathrm{CHO}$ HCP standard concentration over logarithmic scales. 
from the 4-parameter fit standard curve equation of colorimetric and luminescent ELISA, which represent the standard concentrations where the signal response is $\sim 1 / 2$ of the maximum signal response, are 105 and 446 respectively, indicating that luminescent detection has a much broader range. To assist the direct comparison between the two detection methods, relative signal response or signal/noise ratio of each standard was also plotted against its concentration on logarithmic scales (Figure 2B). Luminescent detection showed significant higher signal/noise ratio at all standard concentrations and its standard curve has steeper slope than colorimetric method. As shown in Table 1, the absorbance values at $450 \mathrm{~nm}$ (A450) generated by colorimetric method ranged from 0.075 to 0.561 at standard concentrations from 0 to 2000 $\mathrm{ng} / \mathrm{ml}$; in contrast, light emissions $(425 \mathrm{~nm})$ from luminescent method have a reading from 2461 to 64780 at the same standard range. At 2000 $\mathrm{ng} / \mathrm{ml}$ standard concentration, luminescent ELISA has a signal/noise ratio of 26.32, which is equivalent to 3.5 fold of the $\mathrm{S} / \mathrm{N}$ for colorimetric ELISA (7.467, Table 1). The mean $\mathrm{S} / \mathrm{N}$ for luminescent detection method $(8.677 \pm 8.736)$ is also significantly higher than the mean $\mathrm{S} / \mathrm{N}$ for colorimetric method $(3.191 \pm 2.348)$ as analyzed by paired $t$-test $(p=0.017, \mathrm{n}=11$, Table 1$)$.

To accurately quantify the HCPs in drug substance, the ELISA standard curves need to be fit using the appropriate mathematical models. Several models are available in SoftMax software that include linear, semi-log, log-log, 4-parameter and 5-parameter fit. The method used to form the calibration curve dictates the working range and overall accuracy of the assay [24]. For broad range CHO HCP standard curves as shown in Figure 2, 4-parameter non-linear regression fit gave the best correlation coefficient of $\mathrm{R}^{2}=0.999$. Using the 4 -parameter fit equation, the concentration of each $\mathrm{CHO}$ HCP standard was back-calculated according to its corresponding A450 or E425 value. The precision and accuracy of the back-calculated concentration was demonstrated by its relative standard deviation calculated from triplicate $(\mathrm{CV} \%)$ and the recovery\% (the ratio of back-calculated concentration to the expected concentration) (Table 2). Using CV\% $\leq 30$ and $70 \leq$ Recovery $\% \leq 130$ as acceptance criteria for accuracy and precision, luminescent ELISA has a working/dynamic range from $0.914 \mathrm{ng} / \mathrm{ml}$ to $666.7 \mathrm{ng} / \mathrm{ml}$, which is 27 fold of that for colorimetric ELISA $(2.743 \mathrm{ng} / \mathrm{ml}$ to $74.07 \mathrm{ng} / \mathrm{ml}$, Table 2). In addition, we assessed the linearity of both colorimetric and luminescent ELISA at various standard concentration ranges using several mathematic models and determined their linear range with a log-log fit curve. For luminescent detection, the linear range over logarithmic scales is $0.31-2000 \mathrm{ng} / \mathrm{ml}\left(\mathrm{R}^{2}=0.991\right), 27$ fold of that for colorimetric method $\left(0.91-222.22 \mathrm{ng} / \mathrm{ml}, \mathrm{R}^{2}=0.992\right)$. Moreover, at the $0.91-222.22 \mathrm{ng} / \mathrm{ml}$ standard range, the $\mathrm{R}^{2}$ of luminescent detection for $\log$-log linear fit is 0.998 , showing better linearity than colorimetric method at the same range $\left(\mathrm{R}^{2}=0.992\right.$, Figure 3$)$. The better linearity within the same concentration range was also observed in experiment covering the standard range of $0.781-200 \mathrm{ng} / \mathrm{ml}$, where luminescent

\begin{tabular}{|c|c|c|c|c|}
\hline CHO HCP Standard (ng/ml) & Colorimetric ELISA A450 & Luminescent ELISA E425 & Colorimetric ELISA S/N & Luminescent ELISA S/N \\
\hline 0 & $0.075 \pm 0.003$ & $2461 \pm 265.3$ & 1 & 1 \\
\hline 0.034 & $0.075 \pm 0.004$ & $3124 \pm 140.6$ & 0.993 & 1.269 \\
\hline 0.102 & $0.082 \pm 0.008$ & $3389 \pm 151.6$ & 1.091 & 1.377 \\
\hline 0.305 & $0.086 \pm 0.008$ & $3842 \pm 351.9$ & 1.139 & 1.561 \\
\hline 0.914 & $0.093 \pm 0.004$ & $4924 \pm 216.0$ & 1.244 & 2.001 \\
\hline 2.743 & $0.115 \pm 0.004$ & $7537 \pm 432.9$ & 1.533 & 3.063 \\
\hline 8.23 & $0.153 \pm 0.008$ & $11179 \pm 536.5$ & 2.039 & 4.543 \\
\hline 24.69 & $0.238 \pm 0.018$ & $18228 \pm 335.9$ & 3.17 & 7.407 \\
\hline 74.07 & $0.313 \pm 0.011$ & $28313 \pm 1656$ & 4.17 & 11.51 \\
\hline 222.2 & $0.419 \pm 0.044$ & $39576 \pm 1913$ & 5.577 & 16.08 \\
\hline 666.7 & $0.502 \pm 0.056$ & $49976 \pm 3293$ & 6.683 & 20.31 \\
\hline 2000 & $0.561 \pm 0.034$ & $64780 \pm 3319$ & 7.467 & 26.32 \\
\hline Mean & & & 3.191 & 8.677 \\
\hline Stdev & & & 2.418 & 8.736 \\
\hline \multicolumn{4}{|c|}{$P$ value (paired t-test on mean $S / N, n=11$ ) } & 0.017 \\
\hline
\end{tabular}

Table 1: Comparison of the signal/noise $(\mathrm{S} / \mathrm{N})$ ratios of $\mathrm{CHO} \mathrm{HCP}$ standards from colorimetric ELISA and luminescent ELISA performed in Corning Costar clear 96 -well plate ( $n=3$ for each standard concentration).

\begin{tabular}{|c|c|}
\hline CHO HCP standard $(\mathbf{n g} / \mathrm{ml})$ & \\
\hline & \\
\hline $666.7^{\prime}$ & \\
\hline $222.2^{\prime}$ & \\
\hline $74.07^{\prime, c}$ & \\
\hline $24.69^{\prime, ~}$ & \\
\hline $8.230^{\prime, ~}$ & \\
\hline $2.743^{\prime, ~}$ & \\
\hline $0.914^{\prime}$ & \\
\hline 0.305 & \\
\hline 0.102 & \\
\hline 0.034 & \\
\hline 0 & \\
\hline
\end{tabular}

\begin{tabular}{|c|c|c|c|}
\multicolumn{2}{|c|}{ Colorimetric ELISA } & \multicolumn{2}{c|}{ Luminescent ELISA } \\
\hline$\% C V$ & $\% R e c$ & $\% C V$ & $\% R e c$ \\
\hline 59.97 & 137.66 & 33.69 & $113.15^{\mathrm{b}}$ \\
\hline 105.06 & 160.25 & $26.93^{\mathrm{a}}$ & $87.78^{\mathrm{b}}$ \\
\hline 11.35 & $111.11^{\mathrm{b}}$ & $16.72^{\mathrm{a}}$ & $106.95^{\mathrm{b}}$ \\
\hline $21.80^{\mathrm{a}}$ & $94.67^{\mathrm{b}}$ & $16.75^{\mathrm{a}}$ & $112.54^{\mathrm{b}}$ \\
\hline $17.90^{\mathrm{a}}$ & $118.46^{\mathrm{b}}$ & $4.86^{\mathrm{a}}$ & $100.98^{\mathrm{b}}$ \\
\hline $18.16^{\mathrm{a}}$ & $88.77^{\mathrm{b}}$ & $12.54^{\mathrm{a}}$ & $85.78^{\mathrm{b}}$ \\
\hline 31.8 & $89.84^{\mathrm{b}}$ & $15.76^{\mathrm{a}}$ & $89.39^{\mathrm{b}}$ \\
\hline 13.76 & $84.94^{\mathrm{b}}$ & $15.29^{\mathrm{a}}$ & $72.43^{\mathrm{b}}$ \\
\hline 125.17 & $129.78^{\mathrm{b}}$ & 37.1 & $85.82^{\mathrm{b}}$ \\
\hline 57.38 & 247.84 & $22.16^{\mathrm{a}}$ & 140.18 \\
\hline 75.77 & 171.49 & $27.08^{\mathrm{a}}$ & 267.85 \\
\hline
\end{tabular}

Table 2: Comparison of the precision (\%CV) and accuracy (\%Rec) of back-calculated concentration using 4-parameter fit for colorimetric and luminescent ELISA performed in Corning Costar clear 96-well plate. 
A.

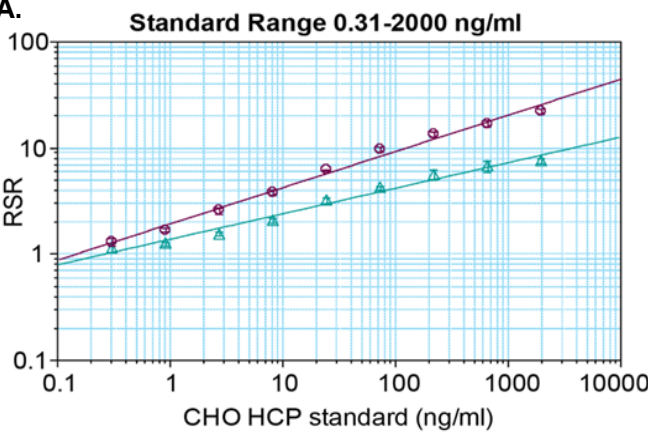

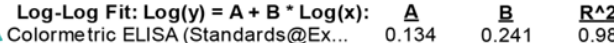
o Lumine scent ELISA (Standards@E...

B.

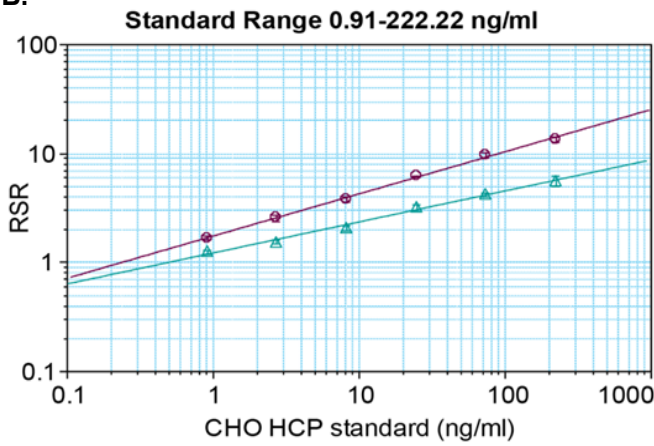

Log-Log Fit: $\log (y)=A+B^{*} \log (x): \quad \underline{A} \quad \underline{B} \quad \underline{R^{\wedge} 2}$ $\begin{array}{llll}\triangle \text { Colormetric ELISA (Standards@EX... } & 0.0818 & 0.285 & 0.992 \\ \circ \text { L Luminescent ELISA (Standards@E... } & 0.237 & 0.387 & 0.998\end{array}$

Figure 3: The linear range and linearity comparison over logarithmic scales between colorimetric ELISA and luminescent ELISA. A. Log-log linearity comparison over the range of $0.31-2000 \mathrm{ng} / \mathrm{ml}$. B. Log-log linearity comparison over the range of $0.91-222.22 \mathrm{ng} / \mathrm{ml}$.

method has a correlation coefficient of $\mathrm{R}=0.999$ for log-log linear fit curve, as compared to $\mathrm{R}^{2}=0.994$ for colorimetric method. In addition to the broader log-log fit linear range and better linearity within the same log-log fit range, luminescent detection also showed better lowend linearity at standard concentrations from 0.034 to $2.743 \mathrm{ng} / \mathrm{ml}$, where the linear correlation has a $\mathrm{R}^{2}=0.992$ in comparison to $\mathrm{R}^{2}=0.961$ for the colorimetric ELISA (Figure 4). Using the low-end linear curve, the concentration of CHO HCPs at $0.914 \mathrm{ng} / \mathrm{ml}$ can be more precisely determined by luminescent ELISA, but not by colorimetric method (Supplemental Table 2).

Within the log-log linear range of $0.91-222.22 \mathrm{ng} / \mathrm{ml}$, the accuracy and precision of both ELISAs were compared. Our results show that within this range luminescent method is significantly more precise (CV\%, $p=0.03$, single tail t-test) than colorimetric method (Table 3). Using the log-log fit curves within this range to back-calculate standard concentrations from 0.03 to $2000 \mathrm{ng} / \mathrm{ml}$, all standard concentrations exhibited comparable or better $\mathrm{CV}$ and recovery values than colorimetric method, which also holds true when back-calculating HCP concentration using 4-parameter fit (data not shown). Despite the standard curve fit methods, luminescent ELISA always has smaller CV values $(\sim 1 / 2)$ than colorimetric method. However, when using log-log fit curve to determine the concentration within this range, luminescent method also exhibit better accuracy (100.65 $\pm 9.12 \%$ recovery) than colorimetric method (102.66 $\pm 18.77 \%$ recovery), although not statistically different ( $p=0.41$, single tail t-test).

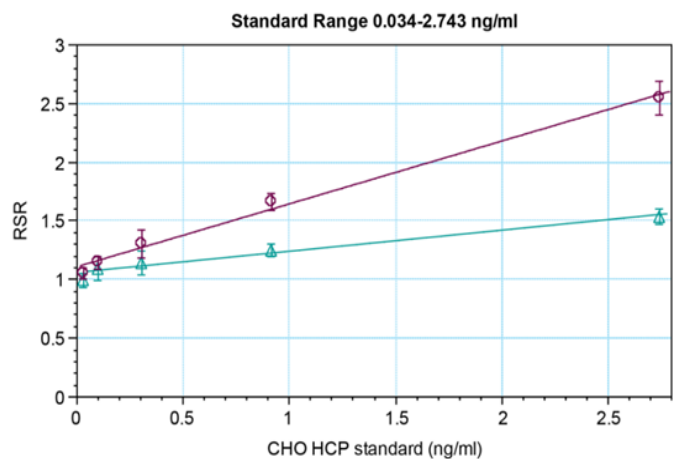

$\begin{array}{cccc}\text { Linear Fit: } \mathrm{y}=\mathrm{A}+\mathrm{Bx}: & \mathbf{B} & \mathbf{B} & \mathbf{R} \wedge \mathbf{2} \\ \triangle \text { Colormetric ELSA (Standards@EX... } & 1.05 & 0.18 & 0.961 \\ \circ \text { Luminescent ELISA (Standards@E... } & 1.1 & 0.536 & 0.992\end{array}$

Figure 4: Low-end linear curves of colorimetric and luminescent ELISA at concentrations ranging from 0.034 to $2.743 \mathrm{ng} / \mathrm{ml}$. The mean signal value \pm standard deviation from triplicate wells was plotted against standard concentration by a linear fit method. Larger variations are observed in colorimetric method at this concentration range. The standard curve is also less linear than luminescent ELISA (R2=0.961 vs. 0.992).

The limit of detection, normally refers to the lower limit of detection (LLOD) is the lowest quantity of a substance that can be distinguished from the absence of that substance (i.e., a blank value) within a stated confidence limit. The lower limit of quantification (LLOQ) is the lowest quantity of a substance that can be accurately quantified. There are a variety of ways to determine the LLOD and LLOQ of an analytical method, here we calculated the theoretical LLOD and LLOQ of ELISA according to the guidelines of ICH-Q2 (R1) using the formula: $\mathrm{LLOD}=3.3 \times \theta / \mathrm{S}$, and $\mathrm{LLOQ}=10 \times \theta / \mathrm{S}$, where $\theta$ is the standard deviation of blank concentration back-calculated from the calibrate curve, $\mathrm{S}$ is the slope of the standard curve. We also determined the experimental LOQ as the lowest and highest standard concentration which can be accurately determined (CV $<20 \%$ and recovery within the range of $100 \pm$ $20 \%)$. Using the log-log fit curve within linear range of $0.31-2000 \mathrm{ng} / \mathrm{ml}$ $\left(\mathrm{R}^{2}=0.991\right)$, the calculated LLOD and LLOQ for luminescent ELISA is 0.245 and $0.816 \mathrm{ng} / \mathrm{ml}$, respectively; in contrast, within its log-log linear range $\left(R^{2}=0.992\right)$, colorimetric method has a LLOD and LLOQ of 0.819 and $2.483 \mathrm{ng} / \mathrm{ml}$, respectively, which is $\sim 2$ times higher than that for luminescent method. When calculated using log-log linear curve fitting in the same range of $0.91-222.22 \mathrm{ng} / \mathrm{ml}\left(\mathrm{R}^{2}=0.998\right)$, luminescent ELISA has a $L L O D=0.365 \mathrm{ng} / \mathrm{ml}$ and $L L O Q=1.216 \mathrm{ng} / \mathrm{ml}$, remaining much lower than that of colorimetric method (Table 4). In addition, lower LLOD and LLOQ is consistently observed in luminescent ELISA from replicated experiments (data not shown) in spite of the methodology used for calculating LLOD and LLOQ, indicating that luminescent detection is indeed more sensitive than colorimetric ELISA for CHO HCP detection and quantification.

One common method to validate the precision and accuracy of ELISA is using the standard curve to measure mAb-A DS with known amount of spike. In our experiments, different amount of CHO HCP was spiked into $5 \mathrm{mg} / \mathrm{ml}$ drug substance and spike recovery was calculated to indicate the accuracy of quantification. Using clear 96-well plate, we observed low recovery on the back-calculated concentration in the spiked samples when the unspiked sample wells are adjacent to the high concentration standards, which lead us to change the assay plate for luminescent ELISA from Costar clear plate to NUNC white opaque plate for assay optimization to avoid cross-interference of luminescence signals. 


\begin{tabular}{|c|c|c|c|c|c|}
\hline & & & \multicolumn{2}{|c|}{ CV\% } & \multicolumn{2}{|c|}{ Recovery\% } \\
\hline & & Colorimetric & Luminescent & Colorimetric \\
\hline & Mean \pm SD & $20.29 \pm 9.12$ & $11.79 \pm 3.67$ & $102.66 \pm 18.77$ \\
\hline Log-log fit & P value (one tail) & 0.03 & & 0.41 \\
\hline & Mean \pm SD & $25.50 \pm 14.27$ & $13.65 \pm 4.58$ & $97.97 \pm 13.59$ \\
\hline & P value (one tail) & 0.04 & $94.68 \pm 14.91$ & 0.35 \\
\hline
\end{tabular}

Table 3: Comparison of mean $\mathrm{CV} \%$ and recovery $\%$ of colorimetric method to that of luminescent method using different fitting method ( $\mathrm{n}=6$, for standards ranging from 0.91 to $222.22 \mathrm{ng} / \mathrm{ml}$ ).

\begin{tabular}{|c|c|c|c|}
\hline Detection method & Log-log fit curve range $\left(\mathrm{R}^{2}\right)$ & LLOD $(\mathrm{ng} / \mathrm{ml})$ \\
\hline Colorimetric & $0.91-222.22 \mathrm{ng} / \mathrm{ml}(0.992)$ & 0.819 \\
\hline Luminescent & $0.91-222.22 \mathrm{ng} / \mathrm{ml}(0.998)$ & 0.365 \\
\hline Luminescent & $0.31-2000 \mathrm{ng} / \mathrm{ml}(0.991)$ & 0.245 \\
\hline
\end{tabular}

Table 4: The LLOD and LLOQ for luminescent and colorimetric ELISA.

\section{The comparison of colorimetric method to luminescent method using conditions optimized for luminescent ELISA in NUNC white opaque 96-well plate}

To avoid cross-talk among adjacent wells, chemiluminescence detection based assays are often carried out in white or black opaque plate. In this study, we also compared the assay performance between luminescent ELISA and colorimetric ELISA using conditions optimized for luminescent ELISA in Nunc white opaque 96-well plate, which provides maximum reflection and low cross-talk. Performing luminescent ELISA in white opaque plate using conditions previously described in clear plate dramatically raised the signal response or relative luminescence unit by 6 to 15 fold. Thus, the reagents concentration used in ELISA assays performed in opaque 96-well plate were re-optimized and finalized as shown in Supplemental Table 1. The assay performance of luminescent and colorimetric ELISA was also compared side-byside using the same amount of assay reagents. The signal/noise ratio, linearity over logarithmic scales, accuracy and precision, as well as the LLOD and LLOQ of both assays were re-assessed.

Under the assay conditions shown in Supplemental Table 1, colorimetric ELISA has a signal response range from 0.056 to 0.121 , the $\log$-log fit linear standard curve has a correlation coefficient $\mathrm{R}^{2}=0.95$, considerably lower than that of luminescent ELISA, which has a signal response range from 4036 to 47731 and a linearity of $\mathrm{R}^{2}=0.998$ (Figure 5).The accuracy and precision of both colorimetric and luminescent ELISA assays were evaluated using the back-calculated standard concentration and the measured spiked DS concentration. For standards, luminescent has significantly lower CV values $(9.76 \pm 8.87$ vs. $28.17 \pm$ 15.96, $p=0.006, \mathrm{n}=8$ ) than colorimetric method, indicating improved precision. For accuracy, the recovery of standards at all concentrations in luminescent ELISA ranges from $94.43 \%$ to $109.73 \%$ (100 $\pm 6.15 \%)$; in contrast standard recovery in colorimetric ELISA ranges from 68.30 to $162.62 \%$, showing much higher variation than luminescent method (Supplemental Table 3). For spiked DS recovery, luminescent ELISA was capable of accurately measure $25 \mathrm{ng} / \mathrm{ml}$ and $5 \mathrm{ng} / \mathrm{ml} \mathrm{HCP}$ spike in 5 $\mathrm{mg} / \mathrm{ml} \mathrm{mAb}$-A drug substance, with much lower $\mathrm{CV}$ values and closer to $100 \%$ recovery than colorimetric method; for $1 \mathrm{ng} / \mathrm{ml} \mathrm{HCP}$ spiked in mAb-A DS (0.2 ppm) recovery, luminescent method was relatively accurate with a $\mathrm{CV}=30.5 \%$ and a recovery $=77.35 \%$, while colorimetric method only showed $39.39 \%$ recovery (Table 5).The LLOD and LLOQ of luminescent ELISA and colorimetric ELISA were calculated according to the standard deviation of blank $(\theta)$ and the slope of calibration curve $(S)$ following the formula $L L O D=3.3 \times \theta / S$ and $L L O Q=10 \times \theta / S$ (Table 6). As shown in Table 6, luminescent ELISA is $\sim 9$ times more sensitivity than colorimetric ELISA when using $0.25 \mu \mathrm{g} / \mathrm{ml}$ biotin-anti-

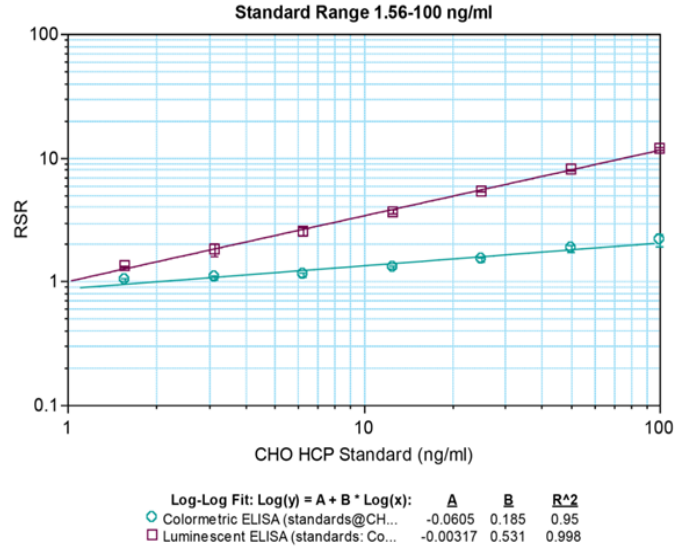

Figure 5: The log-log fit standard curves of colorimetric and luminescent ELISA using conditions optimized for luminescent ELISA and performed in NUNC opaque 96-well plate.

HCP, 1:120,000 dilution of NeutrAvidin-HRP and $50 \mu \mathrm{L}$ of substrate. Additionally, colorimetric ELISA was also repeated in Costar clear 96well plate using concentrations of reagents described above, however, the signal response only ranged from 0.056 to 0.074 at the standards concentrations from 1.563 to $100 \mathrm{ng} / \mathrm{ml}$. The signal response was not sensitive enough to differentiate concentration changes, confirming the much lower sensitivity of colorimetric substrate than luminescent substrate.

\section{Conclusion}

The sensitivity of luminescent detection was compared side-byside with colorimetric detection in a sandwich ELISA format under conditions optimized for either colorimetric ELISA or luminescent ELISA. The performance of both assays including parameters such as signal/noise ratio, linear range and linearity over logarithmic scales, precision and accuracy, as well as LLOD and LLOQ were fully evaluated and compared. Our results show that luminescent detection has enhanced signal/noise ratio, broader linear range on logarithmic scales and better linearity within the same range. In addition, luminescent detection also shows better lower end linearity of its standard curve, which allows the accurate quantification of HCP concentration at as low as $1 \mathrm{ng} / \mathrm{ml}$. Under the same experimental conditions, luminescent ELISA is able to detect and accurately quantify lower amount of HCP than the colorimetric method. Moreover, when calculating HCP concentration from its standard curve, better recovery (closer to 
Citation: Wang F, Driscoll D, Richardson D, Ambrogelly A (2013) The Comparison of Chemiluminescent- and Colorimetric-detection Based ELISA for Chinese Hamster Ovary Host Cell Proteins Quantification in Biotherapeutics. J Bioprocess Biotech 3: 136 doi: 10.4172/2155-9821.1000136

Page 7 of 7

\begin{tabular}{|c|c|c|c|}
\hline & \multicolumn{2}{|c|}{ Colorimetric method } & \multicolumn{2}{c|}{ Luminescent method } \\
\hline & CV\% & Recovery\% & CV\% \\
\hline Unspiked DS & - & & 20.9 \\
\hline $5 \mathrm{ng} / \mathrm{ml}$ spiked DS & 9.8 & 109 & 5.5 \\
\hline $1 \mathrm{ng} / \mathrm{ml}$ spiked DS & 14.4 & 39.39 & 6.3 \\
\hline
\end{tabular}

Table 5: The CV and recovery of spiked DS as back-calculated from the log-log fit calibration curve of luminescent or colorimetric ELISA.

\begin{tabular}{|c|c|c|}
\hline & Colormetric method & Luminescent method \\
\hline LLOD $(\mathrm{ng} / \mathrm{ml})$ & 12.47 & 1.34 \\
\hline $\operatorname{LLOQ}(\mathrm{ng} / \mathrm{ml})$ & 37.78 & 4.06 \\
\hline
\end{tabular}

Table 6: The LLOD and LLOQ of colormetric and luminescent ELISA performed in Nunc white opaque plate using conditions optimized for luminescent ELISA.

$100 \%)$ and lower CV values were observed with luminescent ELISA. Lastly, chemiluminescent ELISA using the Femto substrate doesn't require a stopping step. Chemiluminescent signals can be measured immediately after the addition of substrate and for 10 min after, since the signal will remain at a plateau for that amount of time. In summary, chemiluminescent ELISA proves to be more sensitive than its colorimetric counterpart for mAb-A HCP quantification. Although the experiments were performed using polyclonal antibodies raised against $\mathrm{mAb}$-A null culture $\mathrm{HCP}$, the same principal should apply to the quantitation of HCP in other drug substance samples with minimal assay development work needed. In conclusion, improvement of the HCP ELISA detection limit without compromising assay robustness offers the opportunity to better control HCP clearance during each of the purification steps and reduces the risk of HCP associated immunogenic response in patients.

\section{Acknowledgements}

We thank Xiaoyu Yang and Kimberly May for their support and critical review of this manuscript. The study is funded by the Bioprocess Development Department in Merck Research Laboratories.

\section{References}

1. Brekke $\mathrm{OH}$, Sandlie I (2003) Therapeutic antibodies for human diseases at the dawn of the twenty-first century. Nat Rev Drug Discov 2: 52-62.

2. Leader B, Baca QJ, Golan DE (2008) Protein therapeutics: a summary and pharmacological classification. Nat Rev Drug Discov 7: 21-39.

3. Hudson PJ, Souriau C (2003) Engineered antibodies. Nat Med 9: 129-134.

4. Wurm FM (2004) Production of recombinant protein therapeutics in cultivated mammalian cells. Nat Biotechnol 22: 1393-1398.

5. Nelson AL, Dhimolea E, Reichert JM (2010) Development trends for human monoclonal antibody therapeutics. Nat Rev Drug Discov 9: 767-774.

6. Wang X, Hunter AK, Mozier NM (2009) Host cell proteins in biologics development: Identification, quantitation and risk assessment. Biotechnol Bioeng 103: 446-458.

7. Briggs J, Panfili PR (1991) Quantitation of DNA and protein impurities in biopharmaceuticals. Anal Chem 63: 850-859.

8. Schenauer MR, Flynn GC, Goetze AM (2012) Identification and quantification of host cell protein impurities in biotherapeutics using mass spectrometry. Anal Biochem 428: 150-157.

9. Eaton LC (1995) Host cell contaminant protein assay development for recombinant biopharmaceuticals. J Chromatogr A 705: 105-114.

10. Tscheliessnig AL, Konrath J, Bates R, Jungbauer A (2013) Host cell protein analysis in therapeutic protein bioprocessing - methods and applications. Biotechnol J 8: 655-670.

11. Zhu D, Saul AJ, Miles AP (2005) A quantitative slot blot assay for host cell protein impurities in recombinant proteins expressed in E. coli. J Immunol Methods 306: 40-50

12. Liu N, Brevnov M, Furtado M, Liu J (2012) Host Cellular Protein Quantification. BioProcess International 10: 44-50.

13. Savino E, Hu B, Sellers J, Sobjak A, Majewski N, et al. (2011) Development of an In-House, Process-Specific ELISA for Detecting HCP in a Therapeutic Antibody, Part 2. BioProcess International 9: 68-75.

14. Nicholson P, Storm E (2011) Single-Use Tangential Flow Filtration in Bioprocessing. BioProcess International 9: 38-47.

15. Wang X, Schomogy T, Wells K, Mozier NM (2010) Improved HCP Quantitation By Minimizing Antibody Cross-Reactivity to Target Proteins. BioProcess International 8: 18-24.

16. Gamiz-Gracia L, Garcia-Campana AM, Huertas-Perez JF, Lara FJ (2009) Chemiluminescence detection in liquid chromatography: Applications to clinical, pharmaceutical, environmental and food analysis-A review. Anal Chim Acta 640: 7-28.

17. Lara FJ, Garcia-Campana AM, Velasco Al (2010) Advances and analytical applications in chemiluminescence coupled to capillary electrophoresis. Electrophoresis 31: 1998-2027.

18. Fan A, Cao Z, Li H, Kai M, Lu J (2009) Chemiluminescence Platforms in Immunoassay and DNA Analyses. Anal Sci 25: 587-597.

19. RodaA, Guardigli M (2012)Analytical chemiluminescence and bioluminescence: latest achievements and new horizons. Anal Bioanal Chem 402: 69-76.

20. Dotsikas Y, Loukas YL (2012) Improved Performance of Antigen-HRP Conjugate-based Immunoassays after the Addition of Anti-HRP Antibody and Application of a Liposomal Chemiluminescence Marker. Anal Sci 28: 753-757.

21. Roda A, Manetta AC, Portanti O, Mirasoli M, Guardigli M, et al. (2003) A rapid and sensitive 384 -well microtitre format chemiluminescent enzyme immunoassay for 19-nortestosterone. Luminescence 18: 72-78.

22. Wolter A, Niessner R, Seidel M (2007) Preparation and Characterization of Functional Poly(ethylene glycol) Surfaces for the Use of Antibody Microarrays. Anal Chem 79: 4529-4537.

23. Edgeworth JA, Farmer M, Sicilia A, Tavares P, Beck J, et al. (2011) Detection of prion infection in variant Creutzfeldt-Jakob disease: a blood-based assay. Lancet 377: 487-493.

24. Plikaytis BD, Turner SH, Gheesling LL, Carlone GM (1991) Comparisons of standard curve-fitting methods to quantitate Neisseria meningitidis group A polysaccharide antibody levels by enzyme-linked immunosorbent assay. J Clin Microbiol 29: 1439-1446. 